\title{
Use of dry carbonic acid gas baths to correct human biological age
}

\author{
Viktor Meshchaninov ${ }^{1,2^{*}}$, Denis Shcherbakov ${ }^{1,2}$, Ekaterina Zvezdina ${ }^{3}$, and Ilia Gavrilov ${ }^{1,2}$ \\ ${ }^{1}$ Ural State Medical University, 620028, Yekaterinburg, Sverdlovsk Region, Russian Federation \\ ${ }^{2}$ Institute of Medical Cellular Technologies, 620026, Yekaterinburg, Sverdlovsk Region, Russian \\ Federation \\ ${ }^{3}$ Sverdlovsk Regional Clinical Neuropsychiatric Hospital for war Veterans, 620036, Yekaterinburg, \\ Sverdlovsk Region, Russian Federation
}

\begin{abstract}
Relevance. The physiotherapy method of dry carbonic acid gas baths (DCAGB) mediated through the effects of carbon dioxide $\left(\mathrm{CO}_{2}\right)$ is a minimally invasive treatment and prevention method of many human diseases. However, the $\mathrm{CO} 2$ effect on the rate of human aging has not been sufficiently studied. Therefore, it is relevant to study the effect of dry carbonic acid gas baths (DCAGB) physiotherapy method on peripheral blood indicators and biological age. Purpose. To assess the effect of carbon dioxide in dry carbonic acid gas baths condition on peripheral blood indicators and biological age in patients of different age groups. Patients and methods. An interventional single center controlled clinical trial was conducted on 140 male patients. Within 1 day before the DCAGB sessions course start (10 sessions 40 minutes each) and 1 day after course completion, patients' peripheral blood samples were studied, as well as biological and cardiopulmonal age on the patented method was determined.

Results. DCAGB course reduced biological age in young and middle-aged patients by 5.5 years $(\mathrm{p}<0.001)$, elderly and old patients - by 4.7 years $(\mathrm{p}<0.001)$, elderly and old patients had a decrease in cardiopulmonal bio age by 8.6 years $(\mathrm{p}<0.01)$.

Conclusion. The course of DCAGB sessions slowed the aging rate of the human body from young to old age, which was probably associated with antihypoxic, antitoxic, antioxidant effects of carbon dioxide, as well as possibly with erythropoiesis activation.
\end{abstract}

\section{Introduction}

The problem of organism aging is one of the most pressing issues of the modern science. Currently, there are many different theories about the causes and mechanisms of living organisms aging, indicating that there is no consensus in understanding this complex process $[1,2,3,4]$. In turn, this is the main reason for the lack of universal ways to correct biological age and slow the human aging rate.

\footnotetext{
*Corresponding author: mv-02@yandex.ru
} 
The search for medications to slow the development and manifestation of ageassociated diseases is actively under way [5, 6]. It has been demonstrated that the discovered medications may well be considered heroprophylactic with the effect of slowing the aging rate $[5,7]$. However, this does not give sufficient attention to physiotherapy methods, which are not inferior to medications in their effectiveness.

There is plenty of information in the literature on the group of effective and practical physiotherapy methods based on the influence of different gas regimes on the body [8, 9, $10,11]$. Physiotherapeutic treatment with carbon dioxide $\left(\mathrm{CO}_{2}\right)$ is widespread among these methods. Literature contains information on the role of $\mathrm{CO}_{2}$ in organism metabolism and functions regulation of its systems, on the presence of antioxidant properties of $\mathrm{CO}_{2}$; several mechanisms of its influence on the lipid peroxidation processes in animals and humans are presented etc. $[12,13,14,15]$. At the same time, the role of CO2 in the regulation of aging and biological age change is not sufficiently covered in the literature.

According to certain data, the most effective and low-cost method is the non-invasive therapeutic use of $\mathrm{CO}_{2}$ in the form of dry carbonic acid gas baths (DCAGB) [16]. DCAGB is a method of dermal treatment of $\mathrm{CO}_{2}$ on the patient in a specially equipped box (cabin) and refers to carbogenotherapy [1]. It is known that the beneficial properties of DCAGB can include improvement in oxygen metabolism tissues, acceleration of blood flow and oxygen saturation, immunity strengthening and regeneration activation, improvement of cardiac, pulmonary, nervous systems and endocrine system stabilization, blood pressure normalization, restoration of morpho-physiological skin characteristics in the presence of its diseases, positive effect in correcting excess body mass [17, 18]. It is also known that $\mathrm{CO}_{2}$ can influence the respiratory center of the nervous system contributing to increased respiratory volume, decreased respiratory rate, improved ventilation and gas exchange in the lungs, which will facilitate the blood return to the heart $[19,20]$. However, there is currently little evidence in the literature on the effect of DCAGB on the rate of aging and human biological age. Based on the data available in the literature on the positive effects of $\mathrm{CO}_{2}$ and DCAGB on various human body systems, it is quite relevant to study the action of this physiotherapeutic method on aging and bio-age with the disclosure of possible mechanisms of $\mathrm{CO}_{2}$ heroprotective influence.

\section{Purpose}

To assess the impact of carbon dioxide $\left(\mathrm{CO}_{2}\right)$ in the conditions of dry carbonic acid gas baths (DCAGB) on peripheral blood and biological age in patients of different age groups.

\section{Patients and Methods}

An interventional single center controlled clinical trial was conducted on 140 male patients. Randomly selected patients were divided into two age groups: a group of patients 29 to 55 years of age (young and middle age) and a group of 60 to 89 years of age (elderly and senile age), that corresponded to the classification of human age periods as recommended by WHO.

Inclusion criteria: 29-55 and 60-89 years old male patients with borderline psychoneurological pathology and chronic diseases of internal organs in moderate exasperation or remission phase were involved in the study. Typical basic clinical diagnosis of patients in the study group: coronary heart disease, stage 1-2 hypertensive disease, atherosclerosis, chronic non-specific lung diseases, encephalopathy.

Exclusion criteria: patients with severe diseases expressed in extreme organ and system deficiencies were not involved in the study in the phase of a marked exacerbation of any 
pathology, who have recently suffered severe mental and physical injuries, surgical interventions or other acute and urgent conditions.

Conditions for conducting the study. Outpatient treatment of patients was carried out in the SAHF SO Sverdlovsk regional clinical psychoneurological hospital for veterans of wars, Yekaterinburg.

Description of therapeutic medical intervention. Patients of both age groups in the experiment received a course of transcutaneous exposure in cutaneous resorptive method of carbon dioxide high concentrations using dry carbonic acid gas baths (DCAGB) REABOXSUV" ("Prima-med", Moscow) according to the methodology of P.G. Tsarfis [1, 4, 21]. The course consisted of 10 daily sessions of 40 minutes, on the torso and limbs of a naked patient with a gas mixture of $30-40 \%$ carbon dioxide, humidity of $98 \%, t=30-40 \mathrm{C} 0$ and pressure of 600-800 $\mathrm{mm} \mathrm{Hg}$.

The main outcome of the study was evaluated on studying patients peripheral blood, as well as on the measurement of biological and cardiopulmonal age 1 day before the start of the DCAGB sessions course and 1 day later after its end.

Methods of recording results. Patients peripheral blood collection was performed from the median cubital vein on an empty stomach. To produce red blood cells and plasma, blood with ETDA was centrifuged at $3000 \mathrm{rpm}$ for 15 minutes. Analysis of blood oxygen partial pressure $\left(\mathrm{pO}_{2}, \mathrm{~mm} \mathrm{Hg}\right)$, blood carbon dioxide partial pressure $\left(\mathrm{pCO}_{2}, \mathrm{~mm} \mathrm{Hg}\right)$ and indices of acid-base condition were carried out on the automatic gas analyzer ABL-330 (Radiometr, Copenhagen, Denmark). The number of reticulocytes was counted in peripheral blood smears using Biolam R-11 microscope with immersion lens at 10x90x1.5 magnification. Erythrocyte peroxide fragility (EPF) studies were performed by red blood cell hemolysis measurement in the presence of $3 \% \mathrm{H}_{2} \mathrm{O}_{2}$ and was evaluated in $\%$ as a value reverse to the degree of erythrocyte hemolysis [1]. The LP condition in peripheral blood was assessed by chemiluminescence (CL) of blood plasma induced with hydrogen peroxide on "Lucy3" Anthos Labtec Instruments (Austria) luminometer [22], as well as by the quantity of malondialdehyde (MDA) [23] and dienoic conjugation of higher unsaturated fatty acids (DC) $[23,24]$. With the aim of simplifying the interpretation, the obtained LP values were combined into one integral indicator - the lipid peroxidation coefficient (LPC) $[1,2]$.

Estimation of the number of total lipids and medium-molecular peptides in the patients peripheral blood was carried out using standard kits of reagents using a spectrophotometer "Unico-2802" United Products \& Instruments (USA). Biological (BA) and cardiopulmonal (CPBA) age was determined by our developed and patented methodology $[1,25,26]$. The method of BA and CPBA determination is based on the integral mathematical regression evaluation of a number of human psychophysiological parameters, such as: blood pressure, breath holding time, ventilatory capacity, body weight, accommodation, hearing acuity, static balance, electrocardiogram, pulse wave velocity and opinion health assessment [1].

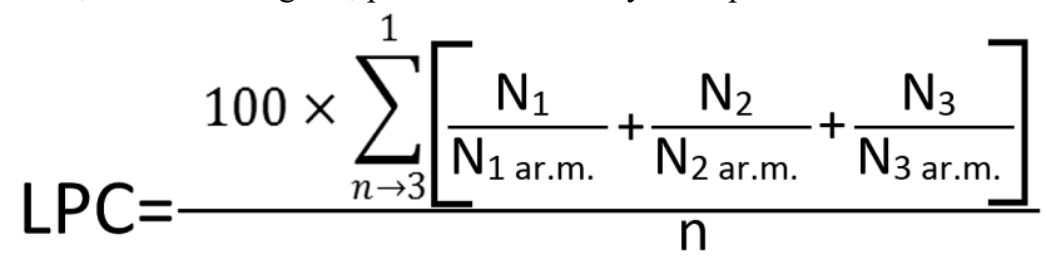

Notes to lipid peroxidation coefficient (LPC) formula:

$\mathrm{N}_{1}$ - chemiluminescent analysis (HL) in an experimental group of patients;

$\mathrm{N}_{2}$ - dienoic conjugation (DC) in an experimental group of patients;

$\mathrm{N}_{3}$ - malondialdehyde (MDA) in an experimental group of patients; 
$\mathrm{N}$ ar.m. - the arithmetic mean of the corresponding LP indicators in the control group of patients,

$\mathrm{n}$ - the number of indicators.

Ethical review. Each patient was familiarized with the conditions of treatment and study with signing informed consent. Since the method of dry carbonic acid gas baths is a common medical treatment technology, the conclusion of the local ethics committee was not required for research.

Methods of statistical data analysis. Research materials were tested for the distribution normality using the Shapiro-Wilk test. If the sample studied had a normal distribution for statistical results processing, the Student parametric t-criterion was applied. Otherwise, Wilcoxon's non-parametric paired t-criterion was applied. $\mathrm{P}<0.05$ was considered the criterion of sufficient reliability of the differences in the comparable averages. The results were processed using Microsoft Office Excel 2016.

\section{Results}

After the effects of the DCAGB course sessions on young and middle-aged patients, in peripheral blood there was a decrease in LP processes intensity with a decrease in LPC by $17.7 \%,(p<0.05)$ and general blood serum lipids value by $18.1 \%(p=0.05)$; on this background, there was significant EPF increase by $54.8 \%(\mathrm{p}<0.05)$, increase of peroxidase activity by $11.0 \%$, decrease of endogenous intoxication processes intensity, $(p=0.05)$, MMP content decrease by $5.8 \%(\mathrm{p}<0.05)$, slight improvement of oxygenation in connection with $\mathrm{pO}_{2}$ by $11.0 \%(\mathrm{p}>0.05)$ and a $3.3 \%$ reduction in excess $\mathrm{pCO}_{2}(\mathrm{p}<0.05)$ (Fig. 1$)$.

After exposure of elderly and senile patients to DCAGB sessions, in peripheral blood there was a repression of the PL processes intensity with a decrease in LPC value by $22.2 \%$ $(p<0.05)$, general blood serum lipids value decrease by $13.2 \% \quad(p=0.05)$, peroxidase intensity increase by $10.3 \%(\mathrm{p}=0.05)$, EPF increase by $23.5 \%(\mathrm{p}<0.05)$, decrease of endogenous intoxication processes intensity in connection with MMP level decrease by $8.1 \%(\mathrm{p}<0.05), \mathrm{pO}_{2}$ oxygenation improvement by $20.0 \%(\mathrm{p}<0.001)$ and blood overalcalinity with $\mathrm{pH}$ value increase from 7.35 to $7.40(\mathrm{p}<0.01)$; hematopoiesis activation reflected in reticulocytes quantity increase by $75 \%(\mathrm{p}<0.05)$ was also noted (Fig. 2$)$.

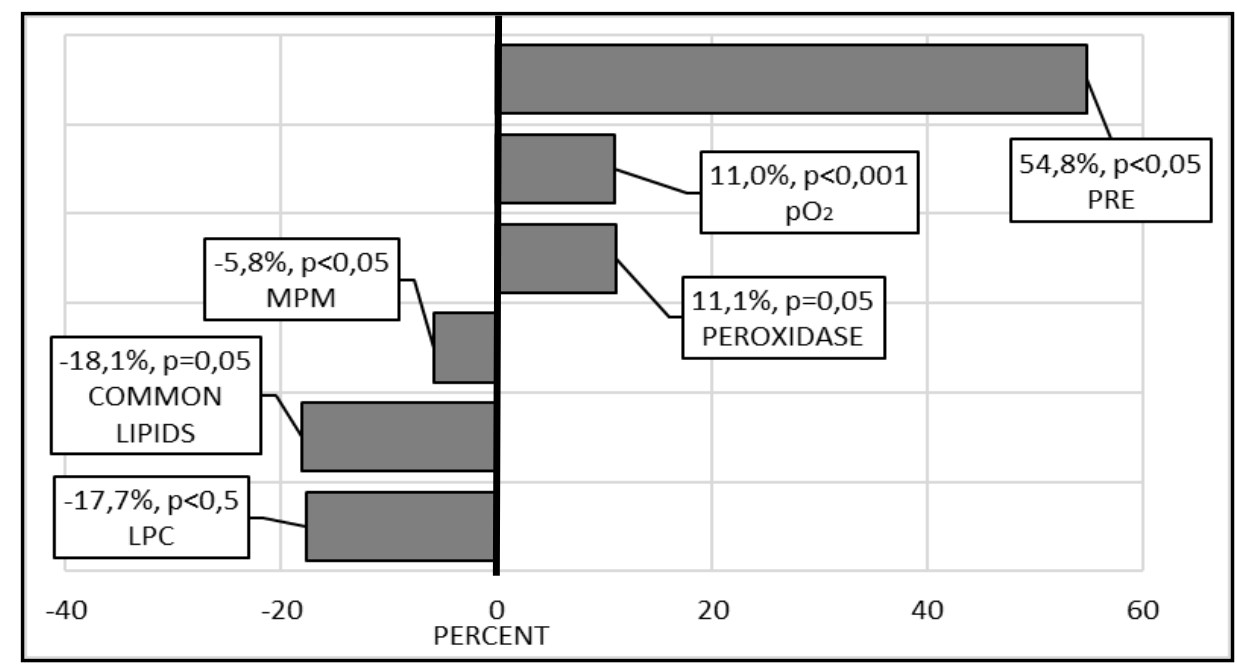

Fig. 1. Changes in the blood of young and middle-aged patients after exposure to a course of dry carbonic acid gas baths sessions (PRE-erythrocyte peroxide resistance, MPM - medium-molecular peptides, LPC - lipid peroxidation coefficient). 


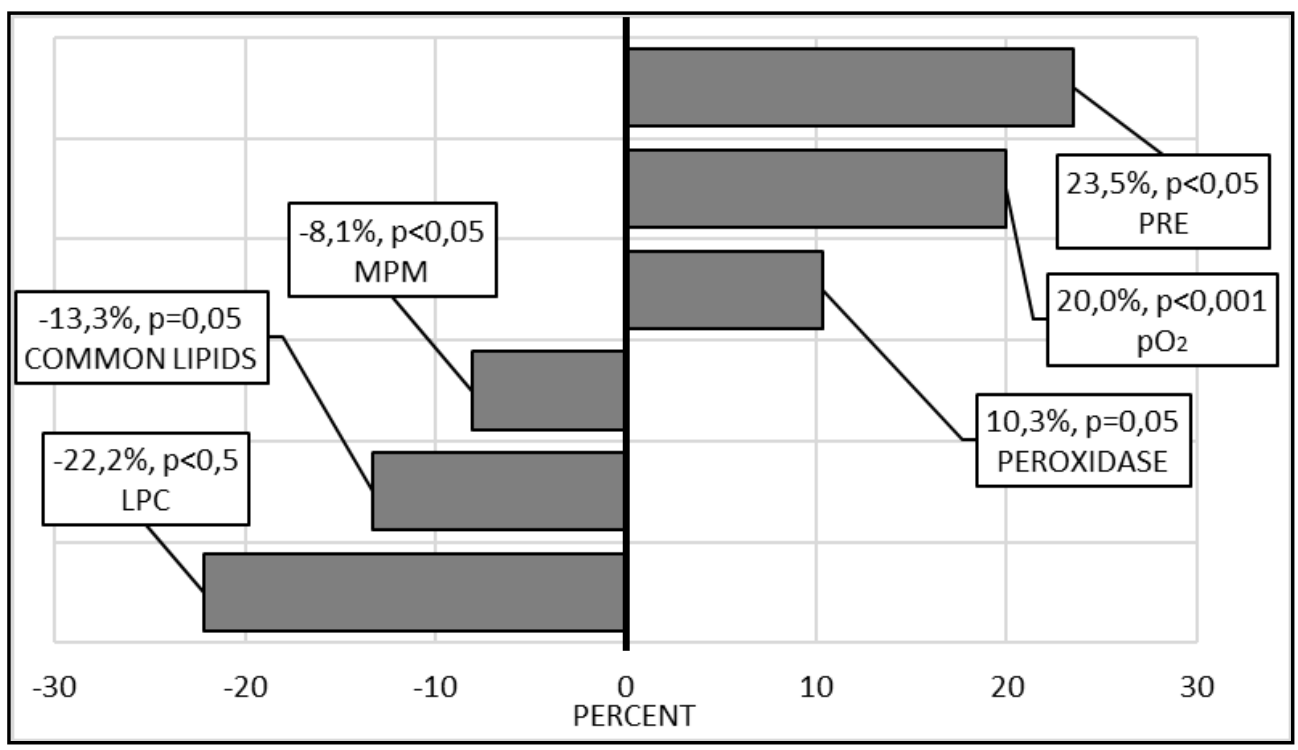

Fig. 2. Changes in the blood of elderly and senile patients after exposure to a course of dry carbonic acid gas baths sessions (PRE — erythrocyte peroxide resistance, MPM — medium-molecular peptides, LPC - lipid peroxidation coefficient)

The DCAGB course in the applied mode has effectively reduced the biological age of young and middle-aged patients by 5.5 years $(p<0.001)$, elderly and senile patients - by 4.7 years $(p<0.001)$; elderly and senile patients also had a decrease in biological cardiopulmonal age $(\mathrm{CPBA})$ by 8.6 years $(\mathrm{p}<0.01)$. Thus, the DCAGB course slowed the rate of organism aging from young to old age, which was probably associated with antihypoxic, antitoxic, antioxidant effects of carbon dioxide gas, as well as possibly with the activation of erythropoiesis (fig. 3).

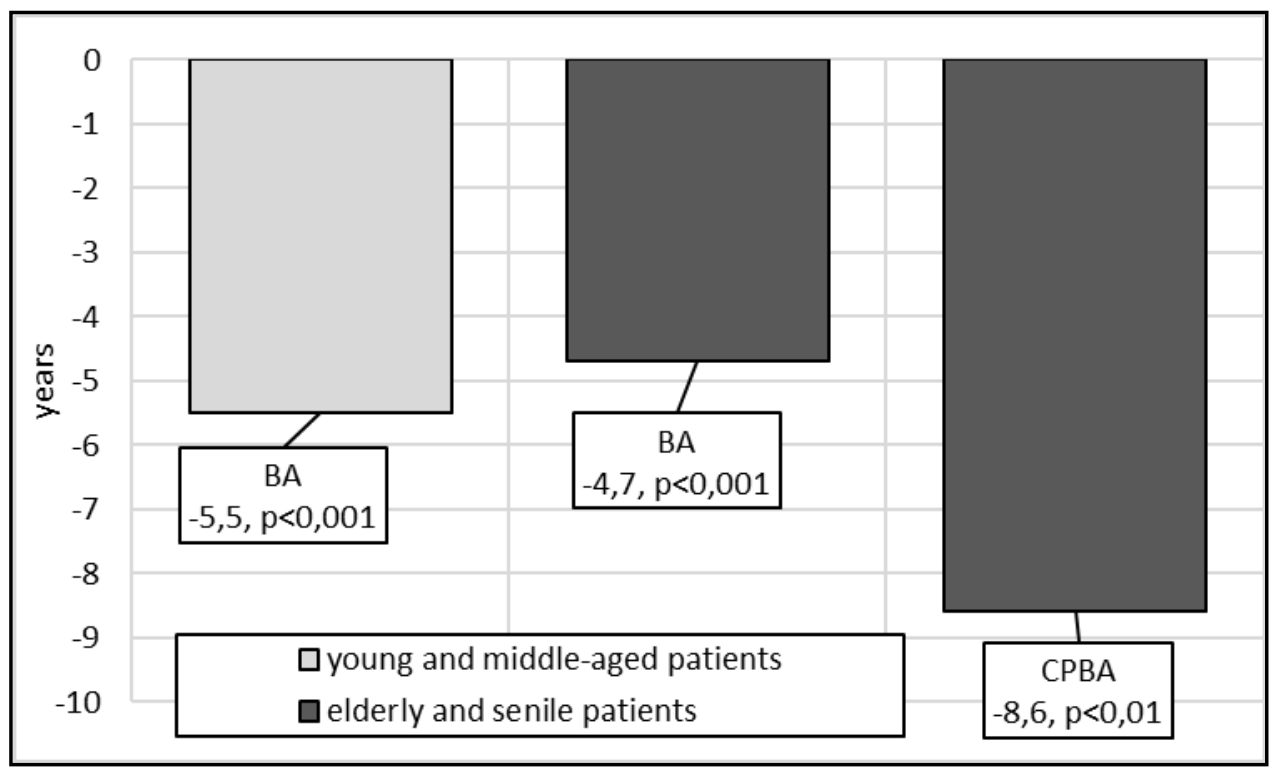

Fig. 3. Changes in biological (BA) and cardiopulmonal (CPBA) age in patients of different age groups after exposure to dry carbonic acid gas baths. 


\section{Discussion}

The DCAGB course influenced patients' peripheral blood in all age groups like an antioxidant, significantly increasing erythrocyte peroxide resistance, which is largely associated with hypolypidemic carbon dioxide effect $[15,14]$. Subsequent to the DCAGB course exposure, most parameters reflecting the level of the LP processes showed significant oppression of the process, additionally elimination of the metabolic acidosis developed during the aging of the organism was observed, as well as increased alkaline reserve and improved peripheral blood oxygenation, which together contributed to the reduction of biological and cardiopulmonal age of patients. Effect of $\mathrm{CO}_{2}$ on cardiopulmonal biological age of elderly and senile patients (Figure 3) might be associated with reduced peripheral vascular resistance or activation of vasodilatation [13] and with regulation of heart rhythm frequency mediated through the effects of $\mathrm{CO} 2$ on the autonomic nervous system [27].

Given that $\mathrm{CO}_{2}$ does not create hypoxic phenomena in the human body [1228,] the leading role of erythropoietin (synthesized in hypoxia) in activation of erythropoiesis during the DCAGB course is out of question. Carbon dioxide had a stimulating effect on hematopoiesis in elderly and senile patients, which is probably related to the following processes:

1) activation of energy and synthetic processes in hematopoietic cells in the state of endogenous hypoxia;

2) strengthening of peroxic and osmotic hemolysis of erytrocytes and participation of its decay products in hematopoiesis stimulation;

3) reducing the severity of endogenous intoxication processes.

\section{Conclusion}

The course of dry carbonic acid gas baths (DCAGB) in all age groups of patients ranging from young to senile age had antihypoxic, antitoxic and antioxidant effect with likely erythroposis activation. These effects resulted in a slowdown in aging processes, resulting in a decrease in the biological and cardiopulmonal age of patients. The DCAGB course is an effective universal heroprophylaxis method of slowing aging and may be recommended for the correction of bio-age in patients of all age groups.

\section{References}

1. V.N. Meschaninov, A.P. Yastrebov Aging, lipid peroxidation and bio-age (Uralsky Sledopyt LLC, Yekaterinburg, 2005)

2. V.N. Meschaninov, D.L. Scherbakov, V.A. Lukash Metabolism of cell structures in aging and stress (USMU, Yekaterinburg, 2017)

3. D. McHugh, J. Gil, Cell Biol, 217(1) (2018)

4. V.S. Myakotnykh, M.N. Torgashov, K.V Egorin, V.N. Meshchaninov et al., Adv Gerontol, 29(4) (2016)

5. A. Moskalev, E. Chernyagina, A. Kudryavtseva, M. Shaposhnikov et al., Aging Dis., 8(3) (2017)

6. A. Moskalev, E. Chernyagina, V. Tsvetkov, A. Fedintsev et al., Aging Cell., 15(3) (2016)

7. A.U. Trendelenburg, A.C. Scheuren, P. Potter, R. Müller et al., Mech Ageing Dev., 180 (2019)

8. I.P. Ivanova, V.M. Nepomnyaschikh, V.S. Shirinsky, V.S. Kozhevnikov et al., Clinical Medicine, 79 (9) (2001) 
9. E.I. Nazarov, Bulletin of Physical Therapy and Resort Science, 22 (2) (2016)

10. E.N. Gerasimenko, V.N. Meshchaninov, U.E. Katireva, E.L. Tkachenko et al., Advances in Gerontology, 5(1) (2015)

11. B.K. Walsh, C.D. Smallwood, Respir Care, 62(6) (2017)

12. E.P. Cummins, M.J. Strowitzki, C.T. Taylor, Physiol Rev., 100(1) (2020)

13. B. Németh, I. Kiss, B. Ajtay, I. Péter et al., In Vivo, 32(6) (2018)

14. N.D. Bunyatyan, S.M. Drogovoz, A.V. Kononenko, A.B. Prokofiev, Vopr Kurortol Fizioter Lech Fiz Kult., 95(5) (2018)

15. F. Eldsouky, H.M. Ebrahim, J Cosmet Laser Ther., 20(5) (2018)

16. V.V. Yezhov, A.Y. Tsaryov, T.E. Platunova, Bulletin of Physical Therapy and Resortology, 23 (2) (2017)

17. V.I., Mizin A.S., Ivashchenko V.V., Yezhov A.Yu., Tsarev et al., Bulletin of Physical Therapy and Resortology, 24 (1) (2018)

18. V.A. Poberskaya, Journal of Restorative Medicine, 85 (3) (2018)

19. V.V. Yezhov, V.I. Mizin, N.A. Severin, A.Yu. Tsaryov et al., Bulletin of Physical Therapy and Resortology, 23 (4) (2017)

20. E.D., Terskikh E.I., Sycheva I.I. Komissarova, Bulletin of Physical Therapy and Resortology, 25 (3) (2019)

21. K.V. Kotenko, A.V. Esipov, V.V. Yamenskov, Voen Med Zh. 337(9) (2016)

22. D.L. Hughes, R.S. Richards, L.A. Lexis, Luminescence, 33(4) (2018)

23. D. Tsikas, Anal Biochem, 524 (2017)

24. J. Frijhoff, P.G. Winyard, N. Zarkovic, S.S. Davies et al., Antioxid Redox Signal, 23(14) (2015)

25. I.V. Gavrilov, V.N. Meschaninov, S.L. Leontiev, Certificate of author's program for computer No. 2012613817/24.04.2012 "Software program "BIOAGE Polinom"

26. I.V. Gavrilov, V.N. Meschaninov, E.L. Tkachenko et al., Patent for invention No. 2617313 / 24.04.2017 "Method of determining biological age in men"

27. Z. Kreska, B. Németh, I. Kiss, I. Péter et al. In Vivo, 32(5) (2018)

28. E.P. Cummins, C.E. Keogh, Exp Physiol, 101(8) (2016) 